\title{
Incidencia del leasing operativo en los estados financieros de las empresas
}

\author{
Incidence of operating leasing in the financial states of companies
}

Incidência do leasing operacional nos estados financeiros das empresas

\section{ARTÍCULO GENERAL}

Fernando Grimaldo Inocente Jacobe

https://orcid.org/0000-0001-6792-3131

fernando.inocente@unmsm.edu.pe

Universidad Nacional Mayor de San Marcos

Universidad Ricardo Palma

Universidad Peruana de las Américas, Lima - Perú
Milagros Sandoval Ypanaque

https://orcid.org/0000-0003-3631-029X

Mili1019@hotmail.com

Universidad Nacional Federico Villarreal Lima - Perú
Domingo Hernández Celis

https://orcid.org/0000-0002-9759-4436

dhernandez@unfv.edu.pe

Universidad Nacional Federico Villarreal

Universidad Norbert Wiener, Lima - Perú

Recibido 07 de Junio 2021 | Arbitrado y aceptado 25 de Agosto 2021 | Publicado en 04 Diciembre 2021

\begin{abstract}
RESUMEN
La investigación tuvo como objetivo determinar en qué medida el leasing operativo incide en el estado de resultados y situación financiera de la compañía minera Mega Exportadora del Perú S.A.C, 2019. La metodología fue de diseño no experimental, de tipo básica con un nivel descriptivo, correlacional y transversal. La muestra del estudio estuvo conformada por 35 colaboradores de la compañía minera Mega Exportadora del Perú S.A.C en el distrito de Magdalena del Mar 2019. Se utilizó la técnica de la encuesta y su instrumento el cuestionario. Se determinó que la herramienta leasing operativo permite el ahorro fiscal y mejora las utilidades de la compañía minera Mega Exportadora del Perú S.A.C en los periodos de estudio. Se concluyó que el leasing operativo incide en el estado de resultados y situación financiera de la compañía minera Mega Exportadora del Perú S.A.C, 2019.
\end{abstract}

Palabras clave: Leasing operativo, Arrendamiento Financiero, Estado de Resultados, Situación Financiera

\section{ABSTRACT}

The objective of the research was to determine to what extent the operating leasing affects the income statement and financial situation of the mining company Mega Exportadora del Perú SAC, 2019. The methodology was nonexperimental design, basic type with a descriptive, correlational level. and transverse. The study sample consisted of 35 employees of the mining company Mega Exportadora del Perú S.A.C in the district of Magdalena del Mar 2019. The survey technique and its instrument, the questionnaire, were used. It was determined that the operational leasing tool allows tax savings and improves the profits of the mining company Mega Exportadora del Perú S.A.C in the study periods. It was concluded that the operating leasing affects the income statement and financial situation of the mining company Mega Exportadora del Perú S.A.C, 2019.

Keywords: Operating Leasing, Financial Leasing, Income Statement, Financial Situation

\section{RESUMO}

O objetivo da pesquisa foi determinar em que medida o arrendamento operacional afeta a demonstração do resultado e a situação financeira da mineradora Mega Exportadora del Perú SAC, 2019. A metodologia foi de design não experimental, tipo básico com nível descritivo e correlacional. e transversal. A amostra do estudo foi composta por 35 funcionários da mineradora Mega Exportadora del Perú S.A.C no distrito de Magdalena del Mar 2019. Foi utilizada a técnica de pesquisa e seu instrumento, o questionário. Foi determinado que a ferramenta de arrendamento operacional permite economia de impostos e melhora os lucros da mineradora Mega Exportadora del Perú S.A.C nos períodos estudados. Concluiu-se que o arrendamento operacional afeta a demonstração do resultado e a situação financeira da mineradora Mega Exportadora del Perú S.A.C, 2019.

Palavras-chave: Leasing Operacional, Leasing Financeiro, Demonstração do Resultado, Situação Financeira 


\section{Introducción}

En mundo globalizado las compañías se han vuelto más competitivas, generando cambios con respecto a la modalidad de aplicar en contabilidad un lenguaje único y estandarizado que permita que la información financiera sea confiable, en especial en las inversiones en bienes de capital, por los grandes desembolsos en su adquisición. Por lo cual, en la mayoría de los casos obligan a las compañías a recurrir a financiamientos, en algunos casos imposibles de acceder a estos préstamos por no contar con liquidez para afrontar dichos pagos, quedando las compañías en procesos productivos muy convencionales, sin innovación tecnológica, estos problemas limitan sus utilidades netas, incluso generan pérdidas que las obliga al cierre de la compañía en el largo plazo.

La globalización de los mercados ha permitido el incremento de la demanda que tienen que satisfacer las compañías, incentivándolos a incurrir en financiamiento, dado que muchas compañías no puedan con sus recursos propios permitir el incremento de su capacidad de producción, para operar por encima de sus condiciones normales como el mercado lo exige. Según Cecilia (2015) la situación que se encuentran muchas compañías cuando "existe la necesidad que tenga que actualizar tecnológicamente sus equipos, maquinarias e instalaciones, para conseguir ser competitivas en la parte de producción y calidad, en el cual debe hacerlo sin afectar la liquidez y solvencia”, son cada vez se vuelve más crítica, por las estrictas condiciones financieras que exigen las instituciones crediticias requisitos que manejan son para protegerse del riesgo de no pago y de esta forma no manejar índices de morosidad elevados que podrían poner el peligro la liquidez y el retorno de sus créditos. (p. 11).

Los profesionales de contabilidad y finanzas de las compañías, se ven en la necesidad de "saber las implicaciones financieras, contables y tributarias que ofrecen las distintas alternativas financieras con la finalidad de optar por la mejor opción," ya que no todas las compañías califican a un crédito o leasing financiero o leasing económico, por diferentes motivos, como la caducidad de los procesos de producción o la insolvencia financiera. Un gran número de compañías, para seguir operando o para aumentar sus ventas, necesitan adquirir activos fijos para seguir operando, conociendo además que estos bienes de capital rápidamente pierden su valor por el crecimiento vertiginoso de la tecnología y la informática, y en su análisis aprecian que la mejora alternativa es acceder al Leasing económico. 
Acorde a lo expresado Ramos (2015) sostiene que: "Dentro de un entorno de competencia global, las instituciones financieras han salido a ofrecer servicios de asesoría empresarial relacionados a nuevos proyectos y alternativas de financiamiento" (p. 1).

Actualmente en el Perú, las compañías están constituidas en su mayoría por Micro y Pequeñas compañías, las cuales no cuentan con "la liquidez necesaria para poder cumplir con las obligaciones con sus proveedores o acreedores, por esto, muchas veces se han visto en la necesidad de obtenerlo mediante el financiamiento de préstamos, pagarés, alquileres y hasta hipotecas con las entidades financieras, con tasas de interés elevadas, la que por consecuencia reflejan un gasto en la organización de manera fortuita" (Ramos, 2015, p. 42).

Las Medinas y las Grandes compañías, generalmente disponen de una mayor liquidez y una mayor situación crediticia para acceder al financiamiento, por lo cual, siempre busca alternativas de financiamiento que le permite al empresario ampliar su capacidad instalada, adquisición de moderna maquinaria y equipos, renovación de flota de transportes, entre otros. Por ello planifican la selección de alternativas de inversión, con sus recursos propias dado que les es más rentable destinarlos al capital circulante o reservarlos a otras finalidades que invertirlos en activos fijos.

Las compañías financieras en el Perú, tienen varias formas de instrumentos crediticios que sirven para el desarrollo de sus proyectos. Entre los más usados, por su mayor potencial, se encuentran el leasing financiero, el leasing operativo, el leasing internacional, en leasing inmobiliario, entre otros; dado a las ventajas que brinda en la parte financiera, económica y tributaria; sin embargo aún hay empresa, como la compañía minera Mega Exportadora del Perú S.A.C que no optan por dichos instrumentos.

La compañía minera Mega Exportadora del Perú S.A.C, se dedica a la extracción de minerales desde hace más de una década, ha logrado un posicionamiento en el mercado a base de esfuerzo y dedicación. Por las propias operaciones realizadas, la compañía constantemente se encuentra en la necesidad de comprar equipos y maquinaria, como: perforadoras, retroexcavadoras, grúas de perforación, entre otras. La modalidad de obtención de los equipos es mediante créditos a largo plazo; sin embargo, los equipos adquiridos se malogra, no tiene los repuestos necesarios, las garantías no cubren a las maquinaria y otras quedan inservible; mientras que la empresa continúa pagando las cuotas crediticias de los activos fijos a los proveedores. 
De continuar, con la modalidad de financiación de sus maquinarias y equipo, la compañía minera Mega Exportadora del Perú S.A.C tendrá problemas con el cumplimientos de sus obligaciones financieras en el corto y largo plazo; así mismo podría disminuir las producciones programadas en los próximos años, en vistas que las maquinarias no tiene un rendimiento óptimo. Una situación extrema, en el largo plazo, la empresa minera puede quebrar, por una un alto endeudamiento y bajo rendimiento de su capital de trabajo.

En ese contexto, el presente estudio plantea una alternativa de financiamiento, como el leasing operativo, el cual es una opción ventajosa de financiamiento utilizada por las grandes compañías (como ya se mencionó también en párrafos anteriores), como las industriales, mineras, constructoras, entre otras, ya que consigue una financiación del cien por ciento de la inversión a realizar y la disposición inmediata del bien adquirido, pagando las cuotas por períodos, sin estipular en el contrato una "opción de adquisición o compra y desde el ámbito tecnológico reponerlo cada vez que se lancen nuevos modelos tecnológicamente mejores" o más avanzados..

La compañía minera Mega Exportadora del Perú S.A.C, tiene un contexto propicio para adquirir un financiamiento de mediano plazo o largo plazo, que permita adquirir activos fijos de grandes inversiones sin compromisos de compra al final del periodo del contrato.

Problema general: ¿En qué medida el leasing operativo incide en el estado de resultados y situación financiera de la compañía minera Mega Exportadora del Perú SAC, $2019 ?$

Problemas específicos: ¿De qué manera el leasing operativo incide en los gastos e ingresos de la compañía minera Mega Exportadora del Perú SAC 2019?; ¿De qué manera el leasing operativo incide en los activos y pasivos de la compañía minera Mega Exportadora del Perú SAC 2019?;

Objetivo general: Determinar en qué medida el leasing operativo incide en el estado de resultados y situación financiera de la compañía minera Mega Exportadora del Perú S.A.C, 2019.

Objetivos específicos: a) Demostrar de qué manera el leasing operativo incide en los gastos e ingresos de la compañía minera Mega Exportadora del Perú SAC 2019. B) 
Demostrar de qué manera el leasing operativo incide en los gastos e ingresos de la compañía minera Mega Exportadora del Perú SAC 2019.

Justificación. Los resultados ayudarán a las compañías arrendadoras a ver los beneficios de adquirir activos fijos, sin la necesidad de incurrir en el gasto de su compra, tanto a compañías nacionales como internacionales. Y más aún, la flexibilidad de contratos de arrendamiento que le permite al cliente adaptar sus pagos de acuerdo con sus preferencias y flujo de caja, ya que el leasing operativo, está "basado en la valoración del activo y en la capacidad del cliente para generar flujos de caja para cumplir con los pagos, contrario a lo que ocurre con el crédito tradicional, donde la solvencia está determinada esencialmente por sus estados financieros y su historial crediticio" (Ramos, 2015).

\section{Importancia del estudio}

Antecedentes nacionales: Al respecto, Odar (2019) considera que el leasing financiero influye en las cuentas de los estados financieros cuando estas arrienden activos fijos debido a que mejora positivamente la economía de la compañía sin la capacidad de contar con efectivo disponible, solo mediante pagos de los activos fijos arrendados. Por su parte, Quevedo (2019) considera que el leasing financiero es una herramienta de financiación para adquirir activos no tangibles. Asimismo, Saccsara y Saldívar (2019) muestra como la aplicación del leasing en una compañía minera mejora sus estados financieros reflejando en sus ratios financieros con buena salud financiera. Entre tanto, Barrenechea-García Faln, Melchor y Minaya (2017) indican que el acceso al leasing operativo trae consigo ventajas contables y tributarias, pero sobre todo es una oportunidad de acceder a un crédito. En cambio, para Rodríguez (2017) demostró que el leasing financiero permite a la empresa mejores posibilidades para cubrir sus obligaciones en el corto plazo, su capacidad de endeudamiento se vuelve aceptable por lo cual le permite adquirir más contratos leasing. Asimismo, Díaz y Mendoza (2016) demuestran que el leasing financiero es una buena opción para el financiamiento de equipos que se busca adquirir y que se puede pagar con cuotas a largo plazo.

Antecedentes internacionales: Para Vásquez (2018) el contrato de Leasing financiero da una mayor facilidad de acceso a la financiación a mediano plazo para las pequeñas y medianas compañías que se encuentran en crecimiento y necesitan ayuda financiera. Para Obiero (2016) considera que a nivel internacional el leasing financiero es una buena opción de arrendamiento y que este no afecta a su desempeño financiero de las compañías, dados que a la par puede acceder a diferentes modalidades crediticias de 
acuerdo con su planificación de su apalancamiento financiero. En cambio, para Pérez (2016) muestra una evolución favorable del leasing en un país Sudamericano, mostrando además que el leasing operativo ha posibilitado el crecimiento de la industria y sobre todo que las compañías pueden renovar sus equipos con la tecnología de punta sin que eso represente un mayor costo para la compañía. Por otro lado, Salam (2015) muestra como el instrumento financiero del leasing influye en los EEFF, mejorando sus indicadores económicos y financieros como son el ROE y ROA para que las compañías puedan proyectarse y tener una rentabilidad adquiriendo activos a largo plazo y no desprender dinero en un solo acto.

\section{Bases teóricas:}

Leasing operativo: El Leasing, se caracteriza por ser un arrendamiento, entendido de la forma que un arrendador compra un bien de capital y lo cede en uso o un arrendatario, pero la propiedad del activo fijo sigue siendo del arrendador hasta que termine el plazo de arrendamiento, en donde de acuerdo con su modalidad contractual puede pasar o no a posesión del arrendatario (Gastón, 2016; Pérez, 2016). De otro lado, el leasing operativo es una alternativa de financiamiento de las compañías productoras como una práctica comercial para colocar sus productos por las dificultades que presentaron los grandes productores de maquinaria y no represento una modalidad financiera propiamente dicha (Bautista y Cussato, 2017). Por su parte, Apaza (2017) refiere que el leasing operativo es el "arrendamiento de un bien durante un tiempo determinado, pero que puede ser revocado por el arrendatario en cualquier momento, con un previo aviso; también, se proporciona el mantenimiento de ese bien, y reponerlo cada vez que se lancen nuevos modelos tecnológicamente más avanzados” (p. 35). Entre tanto, "El leasing operativo no está regulado, en principio, por el Código civil, sino por diversas leyes especiales (siendo la columna vertebral el D.L. 299). Las cuales se fueron emitiendo por necesidades que la sociedad moderna ha ido teniendo a lo largo de los últimos años" (Canelo, 2017, p.35). En cambio para Arrubla (1990) el Leasing como "surge en Estados Unidos a finales del siglo XIX, constituyéndose una importante actividad para el apoyo del crecimiento industrial. Las primeras compañías de leasing fueron los departamentos comerciales de las mismas compañías industriales, o filiales de ellas, que emplearon para su producción” (p. 203). Las ventajas del leasing operativo según Apaza (2017) son: a) Depreciación acelerada con ahorro de impuesto; b) Contra obsolescencia: protección; c) Tesorería sin oscilaciones severas; d) 100\% de Financiación a tasas fijas del bien; e) 
Control presupuestario bueno; f) Menos costo de financiamiento; g) Beneficios Tributarios (Impuesto a la renta - IGV).

Desde el punto de vista normativo, se tiene que de acuerdo con el Código Civil del Perú; el arrendamiento se da cuando el propietario de un bien cede temporalmente su uso y disfrute a otra persona a cambio del pago de una renta. Popularmente se conoce como alquiler, y se formaliza en un contrato. De otro lado, según el Decreto legislativo 299: Arrendamiento financiero se considera que Arrendamiento Financiero, es un Contrato Mercantil que tiene por objeto la locación de bienes muebles o inmuebles por una empresa locadora para el uso por la arrendataria, mediante pago de cuotas periódicas y con opción a favor de la arrendataria de comprar dichos bienes por un valor pactado. Por su parte según Valles (2019), el Decreto Supremo No 179-2004-Ef-Texto Único Ordenado actualizado de la Ley del Impuesto a la Renta establece que el Impuesto a la Renta grava las rentas que provengan del capital, del trabajo y de la aplicación conjunta de ambos factores. De acuerdo con el Artículo $37^{\circ}$, se considera que a fin de establecer la renta neta de tercera categoría se deducirá de la renta bruta los gastos necesarios para producirla y mantener su fuente. De otro lado en el Artículo $38^{\circ}$, las depreciaciones se aplicarán a los fines de la determinación del impuesto y para los demás efectos previstos en normas tributarias. Asimismo según el Decreto Supremo No 055-99-Ef- Texto Único Ordenado de la Ley del Impuesto General a las Ventas e Impuesto Selectivo al Consumo; el crédito fiscal está constituido por el Impuesto General a las Ventas consignado separadamente en el comprobante de pago, que respalde la adquisición de bienes, servicios y contratos de construcción, o el pagado en la importación del bien o con motivo de la utilización en el país de servicios prestados por no domiciliados. Otorgan derecho a crédito fiscal las adquisiciones de bienes, como los de arrendamiento financiero o el alquiler de estos. Asimismo, se tiene a Ley 28424-Ley del Impuesto Temporal a los Activos Netos es un tribut6os que afecta al patrimonio, que grava los activos netos como manifestación de capacidad contributiva. Puede utilizarse como crédito contra los pagos a cuenta y de regularización del Impuesto a la Renta.

En el aspecto contable se tiene a la NIIF 16: Arrendamientos: La norma establece los principios para el reconocimiento, medición, presentación e información a revelar de los arrendamientos. (Ministerio de Economía y finanzas, [MEF, 2020). Arrendamientos operativos: El NIIF 16, revela que un arrendador reconocerá los pagos por arrendamiento procedentes de los "arrendamientos operativos como ingresos de forma lineal o de 
acuerdo con otra base sistemática. El arrendador aplicará otra base sistemática si ésta es más representativa de la estructura con la que se disminuye el beneficio del uso del activo subyacente." (IFRS, citado en por MEF, 2020). "Para arrendamientos operativos, el ingreso del arrendamiento, revelando por separado el ingreso relacionado con pagos por arrendamiento variables que no dependen de un índice o una tasa" (MEF, 2020). En cambio para la NIIF 9: Instrumentos Financieros: La finalidad de la norma "es establecer los principios para la información financiera sobre activos financieros y pasivos financieros, de forma que se presente información útil y relevante para los usuarios de los estados financieros para la evaluación de los importes, calendario e incertidumbre de los flujos de efectivo futuros de la entidad." (IFRS, citado en por MEF, 2020). De otro lado, la NIIF 7: Instrumentos Financieros: Información a Revelar: "El objetivo de esta NIIF es requerir a las entidades que, en sus estados financieros, revelen información que permita a los usuarios evaluar. Luego también se tiene a la NIC 32: Instrumentos Financieros: El objetivo de esta norma es establecer principios para la presentación de los instrumentos financieros como pasivos o patrimonio neto, así como la compensación de activo y pasivo financiero. Otra norma muy relevante es la NIC 16: Propiedad, planta y equipo: El objetivo de esta Norma es prescribir el tratamiento contable de propiedades, planta y equipo, de forma que los usuarios de los estados financieros puedan conocer la información acerca de la inversión que la entidad tiene en sus propiedades, planta y equipo, así como los cambios que se hayan producido en dicha inversión. También entra en juego la NIC 37: Provisiones, Pasivos Contingentes y Activos Contingentes: El objetivo de esta Norma es asegurar que se utilicen las bases apropiadas para el reconocimiento y la medición de las provisiones, pasivos contingentes y activos contingentes, así como que se revele la información complementaria suficiente, por medio de las notas, como para permitir a los usuarios comprender la naturaleza, calendario de vencimiento e importes, de las anteriores partidas. Y para cerrar lo contable la NIC 1: Presentación de Estados Financieros: Esta Norma establece las bases para la presentación de los estados financieros de propósito general para asegurar que los mismos sean comparables, tanto con los estados financieros de la misma entidad correspondientes a periodos anteriores, como con los de otras entidades.

Estado de Situación Financiera: Según Valles (2019), en el marco de la NIC-1: Presentación de estados financieros; se tiene que el estado de situación financiera incluirá partidas que presenten los siguientes importes: (a) propiedades, planta y equipo; (b) 
propiedades de inversión; (c) activos intangibles; (d) activos financieros; (e) inversiones contabilizadas utilizando el método de la participación; (f) activos biológicos dentro del alcance de la NIC 41 Agricultura; (g) inventarios; (h) deudores comerciales y otras cuentas por cobrar; (i) efectivo y equivalentes al efectivo; (j) el total de activos clasificados como mantenidos para la venta.

Estado de resultados: Valles (2019), considera que una entidad reconocerá todas las partidas de ingreso y gasto de un periodo en el resultado a menos que una NIIF requiera o permita otra cosa. Algunas NIIF especifican las circunstancias en las que una entidad reconocerá determinadas partidas fuera del resultado del periodo corriente.

Hipótesis general: El leasing operativo incide en el estado de resultados y situación financiera de la compañía minera Mega Exportadora del Peru S.A.C, 2019.

Hipótesis especificas: a) El leasing operativo incide en los gastos e ingresos de la compañía minera Mega Exportadora del Perú SAC 2019; b) El leasing operativo incide en los gastos e ingresos de la compañía minera Mega Exportadora del Perú SAC 2019.

\section{Método}

Tipo y Método de investigación: La investigación, según su finalidad, es de tipo descriptiva - correlacional. Es descriptiva porque se caracterizó de forma sistemática cada unos de las variables, con la finalidad de identificar las prevalencias en población y diseñara el objetivos e hipótesis del estudio. (Bernal, 2016). La investigación es correlacional, en vista que la finalidad del estudio es establecer las relaciones entre las variables, donde se mide el grado de variación de un fenómeno con respecto a la variación en otro u otros factores. (Monje, 2011)

Diseño especifico de investigación: El diseño de investigación fue no experimental, ya que en el proceso de obtención de los datos de investigación se realizan sin manipulación de variables o intervención directa de los investigadores. (Bernal, 2016). Según Hernández, Fernández, y Baptista (2014) en los estudios con diseño no experimental no se provoca situación o se crean realidades, las variables independientes ocurren y no se pueden manipular, con lo cual el investigador no tiene un control directo sobre dicha variable. Asi mismo el estudio es es no experimental de tipo tranversal, toda que vez que la información fue recopilado en un único momento. 
La población de estudio estuvo conformada por todos los colaboradores de la Compañía Minera Mega Exportadora del Perú S.A.C., el cual asciende a un total de 38 colaboradores entre administrativos, directivos y personal.

Muestra: La muestra para el especialista Behar (2008), se define como un subgrupo extraído de la población de donde se obtendrá datos que deben ser representativo de la población generalizada. Este subgrupo representativo, en la presente investigación, estuvo conformada por 35 participantes. Así mismo, se utilizó el Muestreo Probabilístico Estratificado, debido que la población que se pretende estudiar se encontró dividida en subgrupos, razón por el cual fue necesario tomar elementos (colboradores) de cada sub grupo (Administrativos, directivos y ersonal) (Arias, 2006). (Ver tabla 3)

Instrumentos de recogida de datos: La técnica utilizada en la recolección de la información fue la encuesta. Según Hernández, Fernández y Batista (2014) se utiliza para recolectar información valida a través de la opinión de la unidad de encuesta para establecer los objetivos o el contraste de las hipótesis. (p. 311).

Técnicas de procesamiento y análisis de datos: "El procedimiento para el análisis de la información es establecer el comportamiento de las variables involucradas en el estudio mediante la formación de una base de datos en Microsoft Excel 2010." En el procesamiento de los datos se usó el software SPSS 25, que permitió obtener las estadísticas descriptivas, las tablas de frecuencias y sus gráficos correspondientes, para una explicación descriptiva y comparativa de los resultados.

Procedimiento de ejecución del estudio: Se solicitó un permiso formal al Gerente General de la compañía para la aplicación de los instrumentos de investigación a los colaboradores seleccionados en la muestra, es decir, para los colaboradores del área contable. Se asegura al Gerente General y autoridades de la compañía que se seguirá con el código ético de las investigaciones según los estatutos de la Universidad Ricardo Palma, en la cual se guarda la confidencialidad de los datos y el uso solamente académico de los mismos.

\section{Resultados}

Datos cuantitativos: La comprobación de la confiabilidad de los instrumentos se realizó mediante la prueba de Alfa de Cronbach, la cual mide la consistencia interna de cuestionario de preguntas cerradas en escala politómica. El resultado del coeficiente de alfa de Cronbach debe ser de 0.7 , con lo cual se asegura una confiabilidad aceptable para 
los instrumentos de investigación. (Celina \& Campo, 2005). Los resultados de la confiabilidad para los instrumentos de investigación, para el cuestionario "Leasing Operativo como Instrumento Financiero" obtiene un valor de alfa de 0.912 y el cuestionario sobre ER Y SF obtiene un valor de alfa de 0.963 , los cuales superan el límite de 0.70 . Podemos establecer que los instrumentos de investigación cumplen el criterio de confiabilidad, por lo cual pueden ser utilizados para toda la población.

\section{Análisis estadístico descriptivo:}

Resultado 1: Sobre el nivel de implementación del leasing operativo: según la percepción de los directivos el $8.57 \%$ tiene un nivel malo, el $37.14 \%$ tiene un nivel regular y el 54.29\% tiene un nivel bueno, en la compañía minera Mega Exportadora del Perú SAC - 2019.

Resultado 2: Sobre el nivel de marco normativo leasing operativo: Los directivos se tiene que el $11.43 \%$ tiene un nivel malo, el $31.43 \%$ tiene un nivel regular y el $57.14 \%$ tiene un nivel bueno, en la compañía minera Mega Exportadora del Perú SAC - 2019.

Resultado 3: Sobre la distribución porcentual del nivel de los contratos del leasing operativo: En los directivos el $8.57 \%$ tiene un nivel malo, el $51.43 \%$ tiene un nivel regular y el $40.00 \%$ tiene un nivel bueno, en la compañía minera Mega Exportadora del Perú SAC - 2019.

Resultado 4: Sobre la distribución porcentual del nivel de resultados y situación financiera: La percepción de los directivos el 8.57\% tiene un nivel malo, el $28.57 \%$ tiene un nivel regular y el $62.86 \%$ tiene un nivel bueno, en la compañía minera Mega Exportadora del Perú SAC - 2019.

Resultado 5: Sobre la distribución porcentual del nivel de ingresos de la compañía minera: La percepción de los directivos se tiene que el $8.57 \%$ es de nivel malo, el $28.57 \%$ cree que es de nivel regular y el $62.86 \%$ lo consideró de nivel bueno, en la compañía minera Mega Exportadora del Perú SAC - 2019.

Resultado 6: Sobre la distribución porcentual de gastos de la compañía minera: Los directivos en un $2.86 \%$ considera que es de nivel malo, el $40 \%$ consideró que es de nivel regular y el 57.14\% cree que es de nivel bueno, en la compañía minera Mega Exportadora del Perú SAC - 2019. 
Resultado 7: Sobre la distribución porcentual del nivel de activo de la compañía minera: Los directivos en un $5.71 \%$ considera que es de nivel malo, el $28.57 \%$ cree que es nivel regular y el $65.71 \%$ los consideró como de nivel bueno, en la compañía minera Mega Exportadora del Perú SAC - 2019.

Resultado 8: Sobre la distribución porcentual del nivel de pasivos de la compañía minera. Los directivos en un 5.71\% lo consideró como de nivel malo, el 37.14\% aseveró que es de nivel regular y el 57.14\% lo consideró como de nivel bueno, en la compañía minera Mega Exportadora del Perú SAC - 2019.

Análisis estadístico inferencial: Prueba de normalidad: H0: La distribución de las variables Leasing Operativo y Estados Financieros son paramétricas. H1: La distribución de las variables Leasing Operativo y Estados Financieros no son paramétricas. "Nivel de confianza: 95\% $(\alpha=0.05)$. Regla de decisión: $\rho \geq 0.05$ se acepta la hipótesis nula. $\rho<0.05 \rightarrow$ se rechaza la hipótesis nula. El resultado de la prueba de normalidad de Kruskall Wallis para las variables de investigación, de donde puede establecer que la variable Leasing Operativo son paramétricas $(\mathrm{p}<0.05)$, y la variable Estados Financieros y sus dimensiones, también son paramétricos ( $p>0.05)$. Con lo cual, para la comprobación de las hipótesis relacionadas a la variable Leasing Operativo y estados financieros se usan pruebas paramétricas (correlación de Pearson).

Comprobación de la hipótesis general: La prueba de correlación de Pearson, nos permite establecer que el Leasing Operativo se relaciona significativamente con los Estados Financieros $(\mathrm{p}<0.05)$ de la compañía minera Mega Exportadora del Perú SAC, 2019. Según el coeficiente de correlación $(r=0.47)$ nos evidencia que existe una fuerte relación directamente proporcional entre el Leasing Operativo con los Estados Financieros, es decir que cuando se mejora los niveles de implementación del Leasing Operativo, se mejora el impacto en los Estados Financieros de la compañía minera Mega Exportadora del Perú SAC - 2019.

Comprobación de la hipótesis especifica 1: La prueba de correlación de Pearson, nos permite establecer que el Leasing Operativo se relaciona significativamente con los gastos e ingresos $(\mathrm{p}<0.05)$ de la compañía minera Mega Exportadora del Perú SAC 2019. Según el coeficiente de correlación $(\mathrm{Rho}=0.705)$ nos evidencia que existe una moderada relación directamente proporcional entre la Leasing Operativo y los gastos e ingreso, es decir que cuando se mejora los niveles de Leasing Operativo, se mejora el 
impacto en los gastos e ingreso de la compañía minera Mega Exportadora del Perú SAC -2019 .

Comprobación de la Hipótesis Específica 2: La prueba de correlación de Pearson, nos permite establecer que la utilización del Leasing Operativo se relaciona significativamente con los activos y pasivos $(\mathrm{p}<0.05)$ de la compañía minera Mega Exportadora del Perú SAC - 2019. Según el coeficiente de correlación $(r=0.716)$ nos evidencia que existe una moderada relación directamente proporcional entre la utilización del Leasing Operativo con los activos y pasivos, es decir que cuando se mejora los niveles de utilización del Leasing Operativo, se mejora el impacto en los activos y pasivos de la compañía minera Mega Exportadora del Perú SAC - 2019.

\section{Discusión}

Los resultados obtenidos en la investigación presentan que: La prueba de correlación de Pearson, establece que el Leasing Operativo incide significativamente en el estado de resultados y situación financiera $(\mathrm{p}<0.05)$ de la compañía minera Mega Exportadora del Perú SAC - 2019. Según el coeficiente de correlación r=0.847, evidencia que existe una fuerte relación directamente proporcional entre el leasing operativo con el estado de resultados y situación financiera. Estos resultados se asemejan a los obtenidos por otros investigadores. Como el caso de Quevedo (2019) quien realizó una investigación sobre la incidencia del leasing financiero en el leaseback de los resultados y situación financiera de la Empresa Perú Rail 2018, encontró una relación con nivel de significancia de 0.03 y una probabilidad de 0.97 de la prueba de Pearson, demostrando la relación entre satisfacción laboral y desempeño.

También, Torres (2018), en su estudio sobre la incidencia que tiene los leasing operativos y financieros sobre la gestión administrativa de las microempresas, encontró, en la prueba de chi cuadrado muestra un valor de 12,930 y una sig. de 0.000 , evidenciando que el desconocimiento del leasign operativo tiene influencia significativa sobre la gestión administrativa de las MYPES.

Los resultados descriptivos demuestran que la implementación del leasing operativo en la Compañía Minera Mega Exportadora del Peru S.A.C, según la percepción de los directivos se tiene que el $8.57 \%$ tiene un nivel malo, el $37.14 \%$ tiene un nivel regular y el 54.29\% tiene un nivel bueno. Resultados distintos a los de Quevedo (2019), quien encontró que el 52\% de los empleados está en desacuerdo con las operaciones del 
leasing financiero que aplica la empresa, el 18\% se mostró totalmente en desacuerdo, el $14 \%$ ni de acuerdo ni en desacuerdo, el $8 \%$ de acuerdo y el $8 \%$ totalmente de acuerdo. Por otro lado, Torres (2018) identificó que las empresas de Villa el Salvador, el 75\% nunca tuvo acceso al leasing financiero y el $25 \%$ pocas veces tuvo acceso.

En cuanto a los estados financieros, según la percepción de los directivos se tiene que el $8.57 \%$ tiene un nivel malo, el $28.57 \%$ tiene un nivel regular y el $62.86 \%$ tiene un nivel bueno, en la compañía minera Mega Exportadora del Perú SAC - 2019. Resultados asociados a los de Rodríguez (2017) quien encontró que leasing incide de manera positiva sobre los resultados económicos de la empresa, mediante el leasing se obtuvo una liquidez corriente de 12.3 en el año 2015 y de 12.6 en el año 2016; así mismo, se observó un aumento de utilidad de $11.3 \%$ (2015) a 12.59\% (2016).

Además, la prueba de correlación de Pearson nos permite establecer que el leasing operativo incide en los gastos e ingresos de la compañía minera Mega Exportadora del Perú SAC 2019. Según el coeficiente de correlación $(\mathrm{Rho}=0.705)$ nos evidencia que existe una moderada relación directamente proporcional entre la Normatividad del Leasing Operativo con los Estados Financieros.

Así mismo, la prueba de correlación de Pearson nos permite establecer que el leasing operativo incide en los activos y pasivos de la compañía minera Mega Exportadora del Perú SAC 2019. Según el coeficiente de correlación $(r=0.716)$ nos evidencia que existe una moderada relación directamente proporcional entre la utilización de Contratos del Leasing Operativo con los Estados Financieros.

La investigación concuerda en sus resultados con Saccsara y Saldívar (2019) en su investigación sobre" NIIF 16: Arrendamientos y su incidencia en los Estados Financieros y en el Impuesto a la Renta en las compañías del sector Inmobiliario en Lima Sur, año 2018" al llegar a la conclusión de que la NIIF 16 - Arrendamientos, incide en los estados financieros y en el impuesto a la renta en las compañías del sector inmobiliario en lima sur, año 2018. En la misma línea Odar (2019) en su tesis sobre "El leasing financiero y su influencia en la situación económica de la compañía de transportes Emptursa S.R.L., Chiclayo 2018", donde determinó que existe una influencia de tipo de leasing en la economía de la compañía Emptursa, específicamente llegó a la conclusión que el leasing influye positivamente en la economía de Emptursa. 
En este sentido considerando los cambios de la NIIF 16, sobre la unificación de criterios en el tratamiento del leasing operativo y el leasing financiero para el financiamiento de activos fijos a largo plazo, la investigación encontró también concordancia con Torres (2018) que en su investigación sobre: "El leasing financiero y su impacto en la situación económica financiera de la compañía Motrix E.I.R.L, 2017 en Pimentel - Perú", llegó a la conclusión de que el leasing financiero presenta un impacto positivo para la compañía "MOTRIX E.I.R.L", mejorando la situación financiera al presentar indicadores de liquidez, gestión, solvencia y rentabilidad favorables por presentar influencias en sus EEFF. De la misma manera también, concuerda con Quevedo (2019) en su tesis sobre "El leasing financiero y el leaseback y su incidencia en los resultados y situación financiera de la compañía PERU RAIL 2018”, quien llegó a la conclusión que ambas operaciones de arrendamiento financiero son beneficiosas y se demuestra la incidencia positiva que se obtuvo en los estados financieros del periodo 2018

Así mismo, la investigación es relevante en su análisis sobre preferir el leasing operativo sobre el leasing financiero concordando con Barrenechea-García, Falen, Melchor y Minaya (2017) que investigaron sobre las "Ventajas del Arrendamiento Operativo frente al Arrendamiento Financiero en el Perú en el 2017en Lima-Perú" los cuales llegaron a la conclusión que las ventajas del arrendamiento operativo son superiores al arrendamiento financiero, dado que resulta fácil postular y calificar a un crédito en comparación cuando solicitaron el crédito en alguna entidad financiera, lo que muestra que es una oportunidad a aquellos que fueron marginados por algún motivo, quizá pequeño.

En lo que respecta a la aplicación del arrendamiento en las compañías, en especial el del arrendamiento económico también se ha podido establecer que según los resultados encontrados en las respuestas de los colaboradores contables y financieros de la compañía Mega Exportadora del Perú SAC su implementación en la compañía era bastante buena, con lo que su conocimiento refleja tener conocimiento sobre la actividad financiera mediante el arrendamiento económico, entrando en desacuerdo con Vásquez (2018) que en su tesis sobre: "El leasing financiero y su aplicación en el Ecuador" encontró el leasing no se ha desarrolla completamente en Ecuador puesto a la inseguridad jurídica de sus normas y por no estar complementado con aspectos contables y financieros que vienen asociados a créditos de esta naturaleza tanto como su registro en los EEFF, así como en 
el reflejo de sus indicadores financieros que son analizados para establecer el crecimiento empresarial de las compañías. Además, el Estado ecuatoriano no ha incentivado esta figura mediante el desarrollo de nuevas políticas para reglar de mejor manera el leasing.

En lo referente a la afectación de los ratios financieros de las compañías la investigación también está de acuerdo con Obiero (2016) que en su investigación sobre: "Effects of Lease Financing on the financial performance of Companies listed on Nairobi Securities Exchange in África”, encontró que el arriendo obtuvo un efecto significativo y relación directa en el ROA obteniendo que la financiación por medio de arrendamiento da lugar a una mejora en el ROA. El autor llegó a la conclusión que el rendimiento monetario de las compañías se ve afectado por el financiamiento del arrendamiento.

Y por último, llega a concordar con Pérez (2016) que en su artículo sobre: "El Leasing Financiero un medio para mejorar los resultados de la industria en Bolivia" quien llegó a la conclusión de que las operaciones de leasing más utilizadas han sido el Leasing Operativo que el Leasing Financiero y el Internacional, por el empresario industrial que ha requerido a su banco o compañía arrendataria bienes de capital para su compañía, con la opción de solicitar otro bien de tecnología más avanzada sin que le implique mayor costo de operaciones, mejorando su industria y producto.

\section{Conclusiones}

Las conclusiones son las siguientes:

a) Se determinó que el leasing operativo para ser presentado en los estados financieros, previamente tiene que ser registrado de acuerdo con el Plan Contable General Empresarial (PCGE) e incluso antes tiene que ser reconocido y medido de acuerdo con las Normas Internacionales de Información Financiera (NIIF), para lo cual es necesario tener la capacitación y entrenamiento necesarios sobre las normas antes indicadas, además de las normas legales y normas tributarias como la Ley del impuesto a la renta y la Ley del Impuesto general a las ventas.

b) Se determinó que el reconocimiento del leasing operativo en las transacciones de la empresa se realiza siempre y cuando conlleven a la obtención de ingresos futuros y sea factible de cuantificar o medir; que para el caso del leasing operativo ambos requisitos se cumplen por cuanto el hecho de tener un activo de capital por esta modalidad es para incrementar las actividades de la empresa que 
de hecho darán los ingresos futuros, con lo cual se umple lo que establecen las Normas internacionales de información financiera para el reconocimiento de una transacción empresarial-

c) Se determinó que el leasing operativo incide, tanto en el estado de resultado como en el estado la situación financiera de la compañía minera Mega Exportadora del Perú S.A.C. - 2019. El cual evidencia, que el leasing operativo es un buen instrumento financiero que permite la utilización de activos en la modalidad alquiler, mediante el pago de cómodas cuotas, en un cronograma de pago establecido. Teniendo un efecto positivo, en el corto plazo, en la liquidez (mayor solvencia) de la compañía, debido a que no se realizan grandes desembolsos de activos corrientes. Así mismo, en los periodos (2018-2019) de análisis se demostró que existe una disminución de las pérdidas de la compañía.

d) Se determinó que el leasing operativo incide en los gastos e ingresos de la compañía minera Mega Exportadora del Perú S.A.C. - 2019. La modalidad de leasing operativo permite reducir los gastos (evita grandes desembolsos en el corto plazo) y mejorar los ingresos (mayor liquidez), así mismo permite obtener ahorro fiscales, dado a la modalidad del contrato y la aplicación de depreciación acelerada, por lo cual hay una tributación menor (disminución del impuesto a la renta) debido a que la cuota de leasing operativo se deduce de impuestos.

e) Se determinó que el leasing operativo incide en los activos y pasivos de la compañía minera Mega Exportadora del Perú S.A.C. - 2019. El leasing operativo genera un efecto positivo en la liquidez de la empresa, toda vez que no se realizará grandes desembolsos para comprar maquinarias. Así mismo, el alquiler de capital se pagará en un periodo de 5 años en cuotas mensuales con una tasa de interés fija, el cual no afecta a las decisiones de inversión futuras; así mismo, los gastos de los periodos serán amortiguados y controlados.

\section{Referencias}

Apaza, M. (2017). Análisis Financiero para la toma de decisiones. Instituto Pacífico.

Arrubla, J. (1990). Contrato de leasing. Revista de la Facultad de Derecho y Ciencias Politicas, 203-252.

Asla, E. (2016). La nueva norma NIIF 16, el arrendamiento cambia. Cinco días. Obtenido de 
https://cincodias.elpais.com/cincodias/2016/01/15/economia/1452888776 57991. $\underline{\mathrm{html}}$

Banco Central de Reserva del Perú. (2011). Glosario de términos económicos. Lima: BCRP. Obtenido de www.bcrp.gob.pe/docs/publicaciones/glosario/glosario-

Barrenechea-García, A., Falen, F., Melchor, G., \& Minaya, H. (2017). Ventajas del Arrendamiento Operativo frente al Arrendamiento Financiero en el Perú en el 2017. [Tesis de Maestría, Universidad Peruana de Ciencias Aplicadas]. Repositorio institucional:

https://repositorioacademico.upc.edu.pe/bitstream/handle/10757/622723/barrenec $\underline{\text { h eag_\%clngel.pdf? sequence }=22}$

Bautista, C. y Cussato, H. (2017). SIGMA - Fondo de Inversión en Leasing Operativo Leasop I. Recuperado de https://www.aai.com.pe/wpcontent/uploads/2020/11/Sigma-Jun-20.pdf

Bernal, C. (2016). Metodología de la investigación para administración y economía. Pearson.

Black's Law Dictionary (. (2016). What is Financial Impact? . The Law Dictionary.

Business Dictionary. (2016). Financial Impact. WebFinance Inc.

Canelo, R. (2017). Nociones sobre el contrato de leasing financiero y sus variantes. Recuperado de https://lpderecho.pe/contrato-leasing-financiero/

Cecilia, M. (2015). El Leasing como herramienta de financiación. Universidad. F.A.S.T. Obtenido de Obtenido de http://redi.ufasta.edu.ar:8080/xmlui/bitstream/handle/123456789/866/2015 CP 0 0 2.pdf?sequence $=1$

Celina, H., \& Campo, A. (2005). Aproximación al uso del coeficiente alfa de Cronbach. Revista colombiana de psiquiatría, 34(4), 572 - 580.

Chóren, M. (2015). El Leasing como herramienta de financiación. Universidad Fraternidad de Agrupaciones Santo Tomás de Aquino.

Díaz, A. y Mendoza, N. (2016). El Leasing financiero y su incidencia en la situación económica y financiera de la Empresa Transportes Pedrito S.A.C., distrito de Trujillo, año 2016. [Tesis de pregrado, Universidad Privada Antenor Orrego]. Repositorio institucional. 
Díaz, O. (2010). Estado actual de la aplicación de las NIIF en la preparación de estados financieros de las compañías peruanas. Contabilidad y Negocios, 5(10), 5-28.

Ferrer, A. (2015). NIC 39 Instrumentos Financieros. Revista Actualidad Empresarial, 125.

Gastón, S. (2016). El arrendamiento financiero y valuación de opciones reales. Rev. Contaduría y Administración. 61, (2): 353-373.

Gitman, L., \& Joehnk, M. (2009). Fundamentos de Inversiones. Pearson Educación.

Gonzalo, J. (2006). Las normas internacionales de información financiera: análisis y aplicación. . Revista Dial net, España, 22-38.

Hernández, R., Fernández, C., \& Baptista, M. (2014). Metodología de la Investigación. (Sexta ed.). McGraw-Hill.

IFRS. (2016). Normas IFRS. Recuperado de https://www.ifrs.org/issued-standards/listofstandards

Leiva, J. (2003). El Leasing y su configuración jurídica, Edit. San Marcos.

López, I. (2015). Historia Del Leasing. Enciclopedia Financiera,.

Maaters, T. (2016). What is a Financial Impact? Estados Unidos: Conjecture Corporation. Estados Unidos:: Conjecture Corporation. Obtenido de http://www.wisegeek.com/what-is-a-financial-impact.htm

Ministerio de Economía y Finanzas (2020). Normas Internacionales de Información $\begin{array}{llll}\text { Financiera } & \text { NIIF } & \text { Recuperado de }\end{array}$ https://www.mef.gob.pe/es/?option=com_content\&language=eses\&itemid $=101380 \&$ lang $=$ es-es\&view $=$ article \&id $=5256$

Monje, C. (2011). Metodología de la investigación cuantitativa y cualitativa: Guía didáctica. Neiva: Universidad Surcolombiana.

Mosquera, D., \& Reyes, L. (2018). Proyecto de investigación leasing operativo como medio de financiamiento para compañia Mexichem S.A. [Tesis de pregrado, Universidad de Guayaquil]. Repositorio institucional.

Obiero, J. (2016). Effects of lease financing on the financial performance of companies listed on Nairobi Securities Exchange. Nairobi. (Tesis de grado), University of Nairobi, kenia.

Obtenido de 
http://erepository.uonbi.ac.ke/bitstream/handle/11295/98650/Obiero_Effects\%20o f\%20Lease $\% 20$ Financing $\% 20$ on $\% 20$ the $\% 20$ Financial $\% 20$ Performance $\% 20$ of $\% 2$ 0Companies\%20Listed\%20on\%20Nairobi\%20Securities\%20Exchange.pdf?seque $\underline{\text { nce }}=1 \&$ is Allowed $=y$

Odar, J. (2019). El leasing financiero y su influencia en la situación económica financiera de la compañia de transportes Emptursa S.R.L., Chiclayo 2018. [Tesis de pregrado, $\begin{array}{lllll}\text { Universidad Señor de } & \text { Stenido }\end{array}$ http://repositorio.uss.edu.pe/bitstream/handle/uss/6331/Odar\%20Delgado\%20Jorg $\underline{\text { e } \% 20 \text { Cecilio.pdf? sequence }=1 \& \text { is Allowed }=y}$

Pérez, W. (2016). El Leasing Financiero un medio para mejorar los resultados de la industria. Fides Et Ratio, Vol. 11, pp. 139-154. Obtenido de http://www.scielo.org.bo/pdf/rfer/v11n11/v11n11_a10.pdf

Pérez, W. (2016). El Leasing Financiero un medio para mejorar los resultados de la industria. Fides Et Ratio, Vol. 11, pp. 139-154. Obtenido de http://www.scielo.org.bo/pdf/rfer/v11n11/v11n11_a10.pdf

Quevedo, M. (2019). El leasing financiero y el leasback y su incidencia en los resultados y situación financiera de la compañía PERU RAIL 2018. . [Tesis de pregrado, $\begin{array}{llll}\text { Universidad } & \text { Ricardo } & \text { Obtenido }\end{array}$ http://repositorio.urp.edu.pe/bitstream/handle/urp/2388/cont t030 74471112 \% $\% 2$ 0quevedo\%20alguiar\%2c\%20marina\%20rosario.pdf? sequence $=1 \&$ isallow ed $=\mathrm{y}$

Ramos, G. (2015). El arrendamiento financiero (leasing) y su incidencia en la gestión financiera en la compañia de transportes Ave Fénix sac del distrito de Trujillo año - 2014. [Tesis de pregrado, Universidad Nacional de Trujillo]. Obtenido de: http://redi.ufasta.edu.ar:8080/xmlui/bitstream/handle/123456789/866/2015 CP 0 $\underline{02 . p d f ? \text { sequence }=1}$

Rodríguez, C. (2017). Leasing y su incidencia en la situación económica y financiera de la empresa constructora Riboca S.A de la ciudad de Trujillo - 2016. [Tesis de pregrado. Universidad Cesar Vallejo]. Repositorio institucional.

Ross, W., Westerfield, R., y Jordan, B. (2010). Fundamentos de Finanzas Corporativas. México: McGraw-Hill/Interamericana. 
Rubio, P. (2007). Manual de análisis financiero, Obtenido de www.eumed.net/libros/2007a/255/

Saccsara, M., \& Saldivar, M. (2019). NIIF 16: Arrendamientos y su incidencia en los Estados Financieros y en el Impuesto a la Renta en las compañias del sector Inmobiliario en Lima Sur, año 2018. [Tesis de pregrado, Universidad Peruana de Ciencias Aplicadas].

Obtenidode https://repositorioacademico.upc.edu.pe/bitstream/handle/10757/635422/saccsara tm.pdf? sequence $=5 \&$ isallowed $=y$

Salam, M. (2015). Effects of Lease Finance on Performance of SMEs in Bangladesh. International Journal of Science and Research (IJSR),, Vol. 2, 367-370. Obtenido de www.ijsr.net/archive/v2i12/mtcxmjezmdi=.pdf

Salam, M. (2015). Effects of Lease Finance on Performance of SMEs in Bangladesh. International Journal of Science and Research (IJSR),, Vol. 2, 367-370. Obtenido de www.ijsr.net/archive/v2i12/MTcxMjEzMDI=.pdf

Torres, C. (2018). El leasing operativo y financiero como alternativa en la gestión financiera para bienes de capital en los micros y pequeñas em presas manufactureras en el distrito de Villa el Salvador-Provincia de Lima en el 2017. [Tesis de pregrado, Universidad Nacional Daniel Alcides Carrión]. Repositorio institucional.

Torres, F. (2018). El leasing financiero y su impacto en la situación económica financiera de la compañía Motrix E.I.R.L, 2017. [Tesis de pregrado, Universidad Señor de Sipán]. Repositorio institucional.

Valles, J. (2018). El Renting. Recuperado de: http://tesis.unsm.edu.pe/bitstream/handle/11458/2637/contabilidad\%20\%20jorge \%20alberto $\% 20$ nacarino $\% 20$ pi $\% c 3 \% b 1 \mathrm{a} \% 20 \mathrm{y} \% 20 \mathrm{jhon} \% 20 \mathrm{keiler} \% 20 \mathrm{v}$ alles $\% 20$ garcia.pdf? sequence $=1 \&$ isallowed=y.universidad-nacional-de-sanmartín-tarapoto

Vásquez, P. (2018). El contrato de leasing financiero y su aplicación en el Ecuador. [Tesis de pregrado, Universidad de los hemisferios]. Repositorio institucional. Zeballos, E. (2014). Contabilidad General - Teoría y Práctica. Impresiones Juve E.I.R.L. 